\title{
Determination of accurate stellar radial-velocity measures ${ }^{\star}$
}

\author{
D. Gullberg and L. Lindegren
}

\author{
Lund Observatory, Box 43, 22100 Lund, Sweden \\ e-mail: lennart@astro.lu.se
}

Received 7 February 2002 / Accepted 25 April 2002

\begin{abstract}
Wavelength measurements in stellar spectra cannot readily be interpreted as true stellar motion on the sub-km s${ }^{-1}$ accuracy level due to the presence of many other effects, such as gravitational redshift and stellar convection, which also produce line shifts. Following a recommendation by the IAU, the result of an accurate spectroscopic radial-velocity observation should therefore be given as the "barycentric radial-velocity measure", i.e. the absolute spectral shift as measured by an observer at zero gravitational potential located at the solar-system barycentre. Standard procedures for reducing accurate radial-velocity observations should be reviewed to take into account this recommendation. We describe a procedure to determine accurate barycentric radial-velocity measures of bright stars, based on digital cross-correlation of spectra obtained with the ELODIE spectrometer (Observatoire de Haute-Provence) with a synthetic template of Fe I lines. The absolute zero point of the radialvelocity measures is linked to the wavelength scale of the Kurucz (1984) Solar Flux Atlas via ELODIE observations of the Moon. Results are given for the Sun and 42 stars, most of them members of the Hyades and Ursa Major clusters. The median internal standard error is $27 \mathrm{~m} \mathrm{~s}^{-1}$. The external error is estimated at around $120 \mathrm{~m} \mathrm{~s}^{-1}$, mainly reflecting the uncertainty in the wavelength scale of the Solar Flux Atlas. For the Sun we find a radial-velocity measure of $+257 \pm 11 \mathrm{~m} \mathrm{~s}^{-1}$ referring to the full-disk spectrum of the selected Fe I lines.
\end{abstract}

Key words. methods: data analysis - techniques: radial velocities - stars: kinematics - open clusters and associations: general

\section{Introduction}

Modern radial-velocity spectrometers permit to measure the absolute wavelength shifts of stellar spectral features to better than $100 \mathrm{~m} \mathrm{~s}^{-1}$ (Udry et al. 1999; Nidever et al. 2002). At this accuracy level the interpretation of the observed spectral shifts in terms of stellar radial velocities is non-trivial, due to many factors such as gravitational and convective shifts, template mismatch, and the ambiguity of the classical radialvelocity concept (Lindegren et al. 1999; Lindegren \& Dravins 2002). Recognising this difficulty, the IAU has adopted a resolution (Rickman 2002) identifying the "barycentric radialvelocity measure" $\left(c z_{\mathrm{B}}\right)$ as the appropriate quantity to be determined by accurate radial-velocity spectrometry. The radial-velocity measure is further explained below; briefly, it is the absolute spectral shift corrected only for the accurately known local effects such as the motion of the observer. It is expressed as an apparent velocity, which for normal stars to first order $\left(\$ 1 \mathrm{~km} \mathrm{~s}^{-1}\right)$ coincides with the classical radial velocity.

In order to apply this new concept in accurate radialvelocity work, it is necessary to review many of the established procedures and to modify, or even abandon, some of them. For instance, the practice of using standard stars or

Send offprint requests to: $\mathrm{D}$. Gullberg,

e-mail: dag@astro.lu.se

* Based on observations made at Observatoire de Haute-Provence minor planets to define a velocity zero point is inconsistent with this aim, except at a superficial accuracy level $\left(\sim 0.5 \mathrm{~km} \mathrm{~s}^{-1}\right.$ for normal stars). In this paper we describe and apply a procedure to derive accurate radial-velocity measures from digital échelle spectra. While there may be many other (and also better) ways to achieve this, we hope that the paper may serve as a practical illustration of the new concept, in addition to providing accurate radial-velocity measures for a number of stars.

The procedure is intended to give results that are reproducible in an absolute sense, i.e. without systematic shifts caused by poorly understood or controlled conditions, such as which spectral lines are being used, which parts of the lines define the central wavelengths, and the definition of wavelength scales at the spectrometer and in the laboratory. The means to achieve these goals are not necessarily consistent with techniques that aim for maximum precision, and our procedure is therefore not optimal e.g. for the search of exoplanets.

The spectra used here were obtained in 1997 with the ELODIE spectrometer at Observatoire de Haute-Provence (Baranne et al. 1996) as part of a larger programme to compare the spectroscopic results with astrometric radial velocities (Dravins et al. 1999b; Lindegren et al. 2000; Madsen et al. 2002) in order to study the line shifts intrinsic to the stars (Dravins et al. 1999a). Radial-velocity measures are derived by means of a special reduction procedure using a synthetic template of $\mathrm{Fe}$ I lines, and an absolute zero point defined by the 
Kurucz et al. (1984) Solar Flux Atlas via observations of the Moon. Results for the Sun and 42 stars are given in Tables 1 and 2.

\section{What is the "true" radial velocity?}

Following the general introduction of efficient CCD detectors in astronomy, digital cross-correlation has become a standard technique for determination of stellar radial velocities. In this method the recorded stellar spectrum, after suitable calibration and normalisation, is correlated with a digital mask, or template, and the maximum of the cross-correlation function (CCF) gives the differential velocity of the stellar spectrum with respect to the template. The template could either be based on a real stellar spectrum (e.g. Latham \& Stefanik 1992; Baranne et al. 1996; Gunn et al. 1996) or a synthetic spectrum (e.g. Morse et al. 1991; Nordström et al. 1994; Verschueren et al. 1999). Largely driven by the search for exoplanets, the technique has been refined to provide precisions reaching $\sim 10 \mathrm{~m} \mathrm{~s}^{-1}$ or better for bright solar-type stars (Baranne 1999; Skuljan et al. 2000). Although much attention has also been given to the accuracy of such measurements, i.e. their proximity to "true" values (Griffin 1999), the present practical limitation to "absolute" radial velocities seems to be in the 100-200 $\mathrm{m} \mathrm{s}^{-1}$ range (Stefanik et al. 1999; Skuljan et al. 2000).

Accuracy implies absence of (significant) systematic errors. In the present context, the most important sources of systematic errors are either instrumental, e.g. flexure and slit illumination effects, or related to properties of the (observed) stellar spectrum itself, i.e. what is often referred to as "template mismatch" effects. With the development of highly stable spectrometers it is not unreasonable to assume that instrumental effects can be eliminated to a high degree, and therefore should not be the limiting factor in a well-designed instrument operated under controlled conditions. Systematic errors from template mismatch are an entirely different matter: they seem to be inevitable unless the object and template spectra are almost identical, i.e. resulting from the same source, or at least the same stellar type, and recorded with the same instrument. For instance, it is generally recognised that the use of a single template for different spectral types is likely to cause a sliding and largely unknown zero-point error along the main sequence, with additional systematics caused by differences in stellar rotation, gravity and chemical composition (Smith et al. 1987; Dravins \& Nordlund 1990; Verschueren et al. 1999; Griffin et al. 2000).

Systematic errors and the problems of template mismatch are however also connected with an even more fundamental issue, namely what we mean by the "true" radial velocity (Lindegren \& Dravins 2002). Usually, there is an implicit assumption that the quantity to determine is the actual lineof-sight component of the space motion of the star, or more precisely of its centre of mass. However, it is well known from solar studies that convection in the photosphere causes a net Doppler shift of moderately strong absorption lines by some $-0.4 \mathrm{~km} \mathrm{~s}^{-1}$ in integrated sunlight (Dravins et al. 1981; Allende Prieto \& García López 1998b; Dravins 1999; Asplund et al. 2000). The analysis of line bisectors in stellar spectra
(Gray 1982; Dravins 1987; Nadeau \& Maillard 1988; Gray \& Nagel 1989; Allende Prieto et al. 1995; Allende Prieto et al. 2002) indicates that similar or even stronger blueshifts can be expected for other spectral types. Hydrodynamic 3D simulations by Dravins \& Nordlund (1990) suggest that the convective shift could be $\simeq-1.0 \mathrm{~km} \mathrm{~s}^{-1}$ for F stars to $\simeq-0.2 \mathrm{~km} \mathrm{~s}^{-1}$ for $\mathrm{K}$ dwarfs. Taking into consideration that the gravitational redshift is expected to be fairly constant $\simeq 0.6 \mathrm{~km} \mathrm{~s}^{-1}$ for a wide range of spectral types on the main sequence, the resulting total shift would be in the range from $-0.4 \mathrm{~km} \mathrm{~s}^{-1}$ for $\mathrm{F}$ stars to $+0.4 \mathrm{~km} \mathrm{~s}^{-1}$ for K dwarfs, with the Sun at around $+0.2 \mathrm{~km} \mathrm{~s}^{-1}$.

While the effects of template mismatch can to some extent be studied by means of synthetic spectra (e.g. Nordström et al. 1994; Verschueren et al. 1999), standard model atmospheres are not yet sufficiently sophisticated, for spectral types significantly different from the Sun, to accurately compute the subtle effects of line shifts and asymmetries caused by photospheric convection (Dravins \& Nordlund 1990). On the contrary, empirical determinations of such shifts might be a powerful diagnostic for the study of dynamical phenomena in stellar atmospheres (Dravins 1999). This requires that the true velocity can be established by other means, which was previously possible only for the Sun. Recent advances in space techniques have however made it possible to determine astrometric radial velocities for some stars (Dravins et al. 1997), and future space astrometry missions could provide accurate non-spectroscopic space velocities for a wide variety of stellar types based on purely geometrical measurements (Dravins et al. 1999b).

Given the many problems related to the definition of an accurate spectroscopic velocity zero point, as well as the possibility to determine stellar radial motions by non-spectroscopic means, it has become necessary to make a strict distinction between the two concepts. On one hand, we have the astrometric radial velocity, which by definition refers to the centre-of-mass motion of the star. On the other, we have a spectroscopically determined quantity, which may be expressed in velocity units although it includes non-kinematic effects such as gravitational redshift, as well as local kinematic effects of the stellar atmosphere. The distinction has led to the definition of the (barycentric) radial-velocity measure discussed below.

\section{The "barycentric radial-velocity measure"}

In order to eliminate ambiguities of classical radial-velocity concepts, Lindegren et al. (1999) proposed a stringent definition which was later adopted as Resolution $\mathrm{C} 1$ at the IAU General Assembly in Manchester (Rickman 2002). This recommends that accurate spectroscopic radial velocities should be given as the barycentric radial-velocity measure $c z_{\mathrm{B}}$, which is the measured (absolute) line shift corrected for gravitational effects of the solar-system bodies and effects of the observer's displacement and motion relative to the solar-system barycentre. Thus, $c z_{\mathrm{B}}$ does not include corrections e.g. for the gravitational redshift of the star or convective motions in the stellar atmosphere, and therefore cannot directly be interpreted as a radial motion of the star.

From its definition it is clear that the radial-velocity measure is not a unique quantity for a given star (at a certain time), 
but refers to particular spectral features observed under specific conditions (resolution, etc.). Lest the radial-velocity measure should become meaningless at the highest level of accuracy, these features and conditions should be clearly specified along with the results (cf. Sect. 7.2).

Let $z_{\mathrm{obs}}=\left(\lambda_{\mathrm{obs}}-\lambda_{\mathrm{lab}}\right) / \lambda_{\text {lab }}$ be the observed shift of a certain spectral feature, or (more usually) the mean shift resulting from many such features in a single spectrum. In order to compute $c z_{\mathrm{B}}$ we need to eliminate the effects of the barycentric motion of the observer and the fact that the observation is made from within the gravitational field of the Sun. Lindegren \& Dravins (2002) give the following formula, which for present purposes is accurate to better than $1 \mathrm{~m} \mathrm{~s}^{-1}$ :

$$
\begin{aligned}
1+z_{\mathrm{B}}= & \left(1+z_{\mathrm{obs}}\right)\left(1-\frac{\Phi_{\mathrm{obs}}}{c^{2}}-\frac{\left|\boldsymbol{v}_{\mathrm{obs}}\right|^{2}}{2 c^{2}}\right)^{-1} \\
& \times\left(1+\frac{v_{\mathrm{LOS}}}{c}\right) .
\end{aligned}
$$

Here $c=299792458 \mathrm{~m} \mathrm{~s}^{-1}$ is the speed of light, $\Phi_{\text {obs }}$ the (positive) Newtonian potential at the observer, $\boldsymbol{v}_{\mathrm{obs}}$ the barycentric velocity of the observer and $v_{\mathrm{LOS}}=\boldsymbol{k}^{\prime} \boldsymbol{v}_{\mathrm{obs}}$ the line-of-sight velocity of the observer. ( $\boldsymbol{k}$ is the unit vector from the observer towards the star, taking into account stellar proper motion and parallax, but not aberration and refraction.) The required radialvelocity measure is the barycentric line shift $z_{\mathrm{B}}$ multiplied by the speed of light; it is thus expressed in velocity units although it cannot readily be interpreted as a velocity at the sub- $\mathrm{km} \mathrm{s}^{-1}$ accuracy level.

The main correction in Eq. (1) is the last factor, caused by the observer's barycentric motion along the line of sight. $v_{\text {LOS }}$ is normally provided to sufficient accuracy by standard reduction softwares (Sect. 5.4). The other correction factor is caused by gravitational time dilation and transverse Doppler effect at the observer, and amounts to

$\left(1-\frac{\Phi_{\mathrm{obs}}}{c^{2}}-\frac{\left|\boldsymbol{v}_{\mathrm{obs}}\right|^{2}}{2 c^{2}}\right)^{-1} \simeq 1+(1.48 \pm 0.03) \times 10^{-8}$,

where $\pm 0.03 \times 10^{-8}$ is the amplitude of periodic variations caused by the ellipticity of the Earth's orbit around the Sun. This amplitude corresponds to a maximum error of $0.1 \mathrm{~m} \mathrm{~s}^{-1}$ in the radial-velocity measure. For the present purpose this factor can therefore be regarded as constant. Equations (1)-(2) define the required transformation from observed line shifts to the barycentric radial-velocity measure.

Because the Doppler effect as well as the barycentric correction is multiplicative in the wavelength $\lambda$, the analysis of lineshifts is best made in the logarithmic domain. We introduce $\Lambda=\ln \lambda$, and use the dimensionless $u$ to designate a small shift in $\Lambda$. The shift can also be expressed in velocity units as $d=c u$. This is related to the usual spectral shift $z$ through $1+z=\exp (d / c)$, which by expansion gives $c z=d+d^{2} /(2 c)+d^{3} /\left(6 c^{2}\right)+\cdots . c z$ and $d$ are therefore alternative ways of expressing spectral shifts in velocity units, differing in the second-order terms, but none of them strictly representing physical velocity $v . d$ has the advantage over $c z$ that the various effects (or corrections) are additive in this variable. The distinction between them disappears, to the nearest $\mathrm{m} \mathrm{s}^{-1}$, for shifts $<17 \mathrm{~km} \mathrm{~s}^{-1}$. Second-order relations are adequate to the same precision for shifts $<600 \mathrm{~km} \mathrm{~s}^{-1}$.

The radial-velocity measure for a star should be referred to the mean epoch of observation, expressed as the barycentric time of arrival $\left(t_{\mathrm{B}}\right)$, i.e. the time of observation $\left(t_{\mathrm{obs}}\right)$ corrected for the Rømer delay associated with the observer's motion around the solar-system barycentre. Neglecting terms due to relativity and wavefront curvature, which together are less than $1 \mathrm{~ms}$ for stellar objects, this correction can be computed as

$t_{\mathrm{B}}=t_{\mathrm{obs}}+\boldsymbol{k}^{\prime} \boldsymbol{r}_{\mathrm{obs}} / c$

(Lindegren \& Dravins 2002), where $\boldsymbol{r}_{\mathrm{obs}}$ is the barycentric position of the observer at the time of observation and $\boldsymbol{k}$ the previously defined unit vector towards the star.

\section{The observations}

ELODIE (Baranne et al. 1996) is an échelle spectrometer physically located in a coudé room at the $1.93 \mathrm{~m}$ telescope at Observatoire de Haute-Provence (OHP). For this programme, the spectrometer was fed via one optical fibre from the Cassegrain focus. The instrument $F W H M$ is $\simeq 7.2 \mathrm{~km} \mathrm{~s}^{-1}$, corresponding to a resolving power of $R \simeq 42000$. The present observations were made 1997 in two separate runs on February 18-23 and October 15-23. The campaign targeted mainly stars in the Hyades and Ursa Major open clusters, but also included a set of IAU radial-velocity standards, some low-metallicity stars, Procyon and 51 Peg. Lunar spectra were also obtained for the purpose of calibrating the absolute wavelength scale.

The strategy during the observations was to have as good signal-to-noise ratio $(S / N)$ as possible, in order to allow also weak lines to be used for the extraction of differential velocity information (Gullberg 1999). With the gain factor used, $2.65 \mathrm{e}^{-} \mathrm{ADU}^{-1}$, the maximum $S / N$ is about 300 before nonlinearity and saturation effects occur in the most flux-rich orders.

The normal operation of ELODIE, when used as a radialvelocity machine, is to obtain spectra of modest $S / N \simeq 50$ in short exposures, with Th-Ar calibration spectra obtained simultaneously occupying the inter-order spaces of the CCD image. For the present programme it was considered important to avoid any possible light or charge leakage from the Th-Ar exposure; therefore separate Th-Ar exposures were made, leaving the inter-order space of the stellar spectra empty. Several such calibration exposures were obtained during each night, ideally between each stellar exposure.

Observations of the Moon were needed to derive an absolute wavelength scale and to correct for any long-term instability of the instrument, in particular between the February and October sessions. During the lunar observations the intended target was a well-defined crater or bright surface near the selenographic centre, although this turned out to be difficult to achieve in practice. Fortunately, even an offset by several arcmin would not cause any significant zero-point error (Sect. 5.4). 


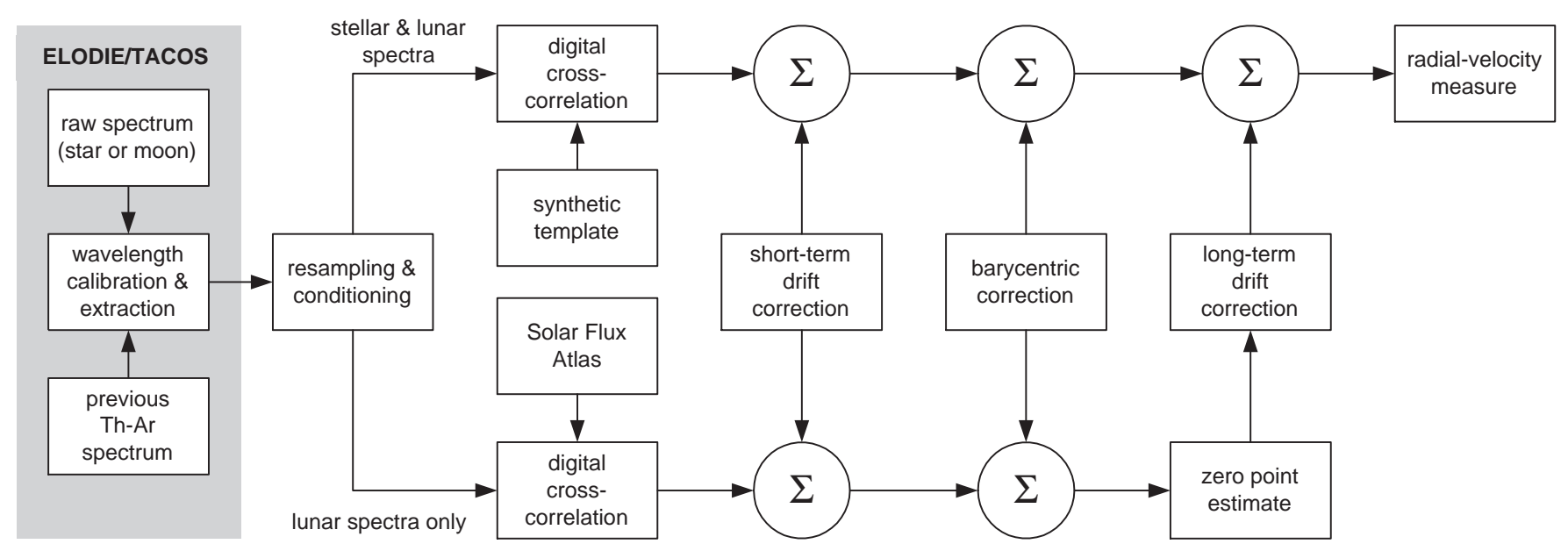

Fig. 1. This flowchart outlines the reduction process described in the text. The grey box contains processes that are included in the ELODIE software package TACOS. All spectra of stars and the Moon are piped through the upper branch of processes, and thus receive identical treatment. The lunar spectra are also passed through the lower branch in order to determine the long-term drift correction which effectively defines the zero point of the final radial-velocity measures.

\section{Data reductions}

The basic steps of the data reduction method are illustrated in Fig. 1. The successive steps, represented by boxes in the diagram, are explained in the following subsections.

The shaded box in Fig. 1 contains steps that are performed by the ELODIE software TACOS (Queloz 1996). Using the most recent Th-Ar exposure, TACOS computes a two dimensional Chebychev polynomial which maps each pixel to a wavelength. Using this map, the two-dimensional échelle spectrum is reduced to one-dimensional spectra on a nominal wavelength scale which therefore is based on the previous $\mathrm{Th}-\mathrm{Ar}$ spectrum.

After resampling and conditioning (Sect. 5.1) the spectrum is digitally correlated with a synthetic template (Sect. 5.2), giving the spectral shift relative to the nominal wavelength scale. This is then corrected for short-term drift (Sect. 5.3), barycentric motion (Sect. 5.4) and long-term drift (Sect. 5.5). In this part of the reductions, shown by the upper branch in Fig. 1, all spectra receive exactly the same treatment, resulting in our estimated radial-velocity measures. For the lunar spectra it gives the radial-velocity measure of the Sun. In the lower branch of the diagram, used only for the spectra of the Moon, long-term drift (or the absolute zero point) is determined through crosscorrelation with the Solar Flux Atlas (Kurucz et al. 1984); this defines the final wavelength scale.

The ELODIE software also provides radial-velocity determinations based on either or both of two standard ELODIE templates, corresponding to FOV and KOIII stars (both containing box-shaped lines derived from model atmosphere spectra, see Baranne et al. 1996). These velocities are not further discussed in this paper, although they did provide a useful consistency check of our own procedure.

\subsection{Resampling and conditioning of the spectrum}

Before correlating, all 67 orders extracted by the ELODIE software are combined into a single one-dimensional spectrum, normalised, flipped, resampled and windowed. This conditioning of the spectrum is illustrated in Fig. 2.

The purpose of the normalisation is to equalise the flux distribution, so that the relative weights of different spectral regions is independent of arbitrary factors such as the wavelength response of the spectrometer (cf. Sect. 7.3). Tungsten exposures are used to remove the main part of the variation within each order. "Continuum" points are then identified and used to normalise the flux values to the interval $[0,1]$. Data from the different orders are combined into a single sequence of flux/wavelength pairs by removing the blue ends of overlapping orders.

Both the observed data set and the template are then flipped and resampled with a constant step of $\Delta \Lambda=5 \times 10^{-7}$ in $\Lambda=\ln \lambda$. Using a logarithmic wavelength scale renders the Doppler shift independent of wavelength (cf. Sect. 3). The chosen resampling step, corresponding to a velocity step of $\simeq 150 \mathrm{~m} \mathrm{~s}^{-1}$, is more than adequate to preserve all spectral information (it gives $\simeq 50$ steps across the instrumental $F W H M$, and $\simeq 20$ steps per pixel), and allows accurate sub-step centroiding by simple interpolation (Eq. (5)). The resulting resampled data sequence is denoted $\left(\Lambda_{i}, f_{i}\right), i=1 \ldots n$. To avoid spurious effects from the edges of the stellar spectrum and template, the flux data are furthermore multiplied with a flat-topped cosine window function $0 \leq w_{i} \leq 1$, such that the outermost $5 \%$ at each end smoothly taper off to zero.

\subsection{Digital cross-correlation}

The ELODIE radial-velocity measurements are normally based on synthetic templates derived from stellar atmosphere 


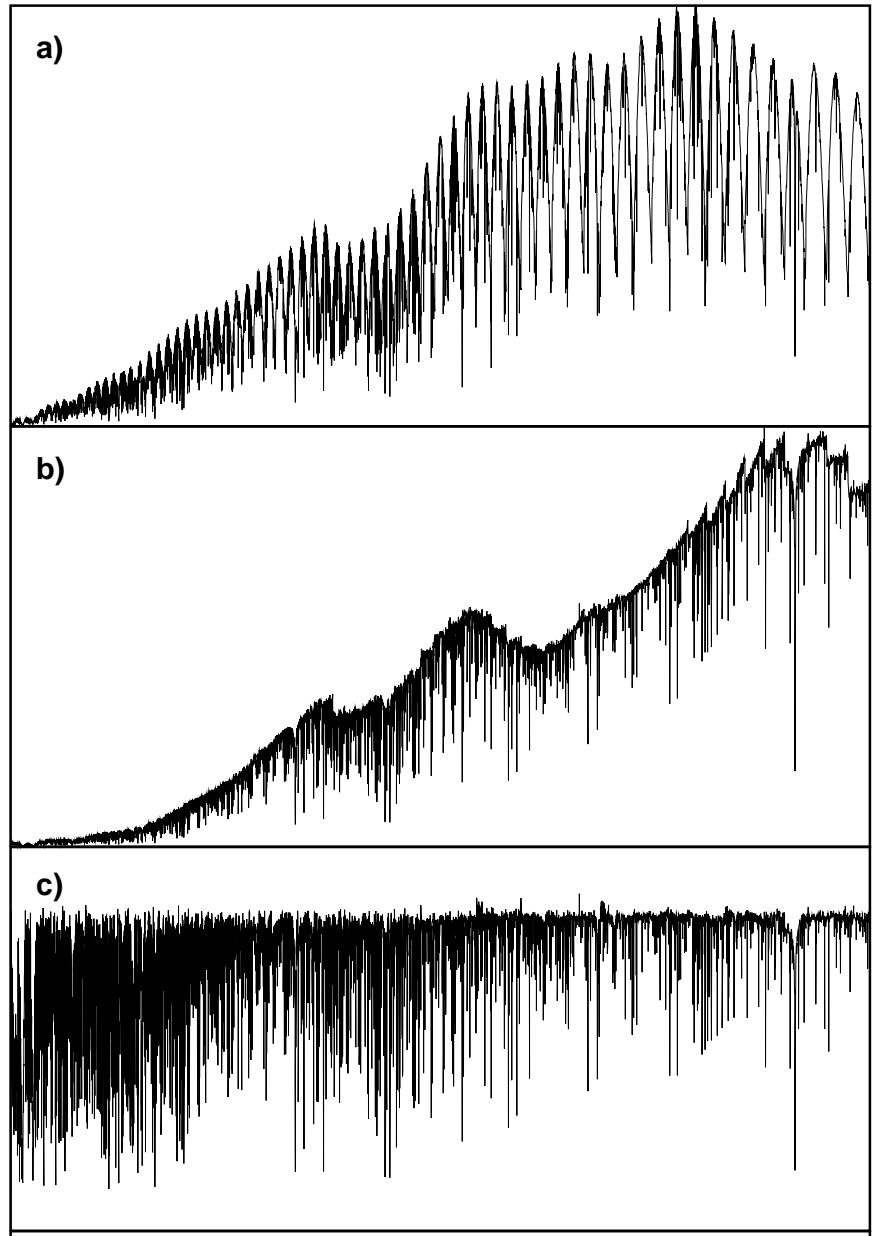

d)

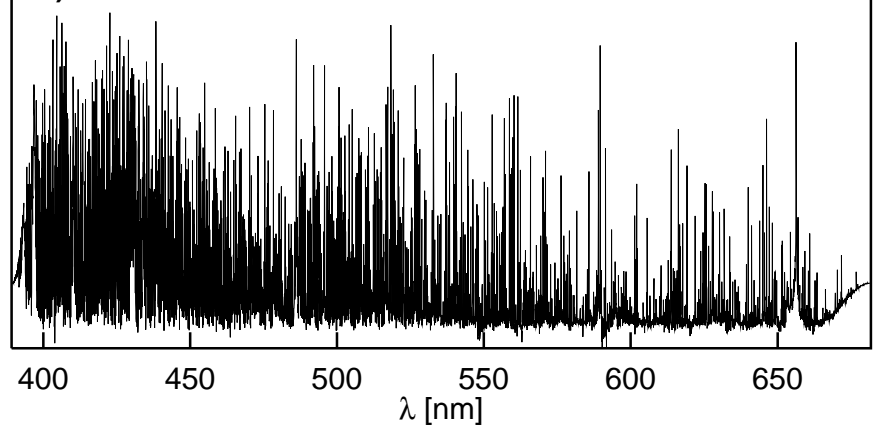

Fig. 2. A series of diagrams illustrating the conditioning of an observed spectrum before it is correlated with the template. a) The raw one-dimensional spectrum versus pixel number, as extracted from the CCD image. Note the characteristic parabolic envelope of each spectral order. b) The wavelength scale has been set and the gross variation within each order removed using calibration observations of a tungsten lamp. c) The spectrum has been normalised through division by an estimate of the continuum intensity. d) The spectrum has been inverted, its average subtracted, and the windowing function applied to taper off the ends. This is what goes into the digital correlation. The Solar Flux Atlas, used as a template for the observations of the Moon, is treated similarly, going from c) to d).

models. We have instead chosen to use a template based only on $1340 \mathrm{Fe}$ I lines, for which very accurate laboratory wavelengths exist (Nave et al. 1994). The lines were selected in the wavelength region 400-680 $\mathrm{nm}$ through comparison with the Allende Prieto \& García López (1998a) catalogue of solar lines. The majority of the lines are of quality grade ' $A$ ' in the list by Nave et al. implying wavenumber uncertainties below $0.005 \mathrm{~cm}^{-1}$ or $75 \mathrm{~m} \mathrm{~s}^{-1}$ at $\lambda=500 \mathrm{~nm}$, and have upper levels of moderate excitation $(<6.5 \mathrm{eV})$, for which pressure-dependent shifts in the laboratory wavelengths should be small. The template was built by unit height Gaussian functions having a constant $F W H M$ of $W=5 \mathrm{~km} \mathrm{~s}^{-1}$. For the lunar observations, we also use the Solar Flux Atlas (Kurucz et al. 1984) as template.

Let $s_{i}=w_{i} f_{i}, i=1 \ldots n$ be the stellar spectrum resulting from the conditioning described above, and $t_{i}$ the similarly conditioned template. Both data sets are equidistantly sampled in $\Lambda=\ln \lambda$ with step $\Delta \Lambda$. The cross-correlation function (CCF) is computed as

$g_{j}=\sum_{i} s_{i} t_{i-j}, \quad j=0, \pm 1, \pm 2 \ldots$

These values can be regarded as discrete points of the continuous CCF $g(u)$ for $u=j \Delta \Lambda$. The cross-correlation process should find $\hat{u}$ such that $g(\hat{u})$ is the global maximum of the CCF. We do this by fitting a parabola to the three points nearest to the maximum of the discrete CCF and computing the point where the analytical derivative of the parabola is zero $\left(g^{\prime}(u)=0\right)$. If $g_{j}$ is the highest point in the discrete CCF $\left(g_{j-1} \leq g_{j}>g_{j+1}\right)$, then

$\hat{u}=\left[j+\frac{\frac{1}{2}\left(g_{j+1}-g_{j-1}\right)}{2 g_{j}-g_{j-1}-g_{j+1}}\right] \Delta \Lambda$.

Multiplying $\hat{u}$ with the speed of light gives the shift $d_{\mathrm{N}}$ expressed in velocity units. The subscript " $\mathrm{N}$ " indicates the nominal wavelength scale obtained from the current Th-Ar calibration.

The uncertainty of $\hat{u}$ from photon and readout noise in the CCD image, $\sigma_{\hat{u}}$, is estimated according to Eq. (A.3) derived in the appendix. In velocity units $\sigma_{\mathrm{N}}=c \sigma_{\hat{u}}$ ranges from $2 \mathrm{~m} \mathrm{~s}^{-1}$ to several $100 \mathrm{~m} \mathrm{~s}^{-1}$ for the observations reported in Table 2; the median value is $13 \mathrm{~m} \mathrm{~s}^{-1}$.

A short remark should be made concerning our method to compute the maximum of the digital CCF. An alternative procedure described in the literature (e.g. Murdoch \& Hearnshaw 1991; Gunn et al. 1996; Skuljan et al. 2000) is to fit a Gaussian, or some other suitable function, to a wider part of the correlation peak. We believe that this procedure is inappropriate from the viewpoint of statistical estimation theory in our case, or when model-atmosphere spectra are used as templates. Maximising the CCF is equivalent to minimising the $\chi^{2}$ or some similar function representing the goodness-of-fit between the template and spectrum, and it is then the extreme point of the objective function that should be sought ${ }^{1}$.

1 This remark does not apply to techniques where the spectrum is optically correlated with a hardware mask (e.g. Griffin 1967; Baranne et al. 1979): in that case the use of a wider part of the correlation peak helps to reduce the effects of the shot noise affecting the individually measured $\mathrm{CCF}$ points. 

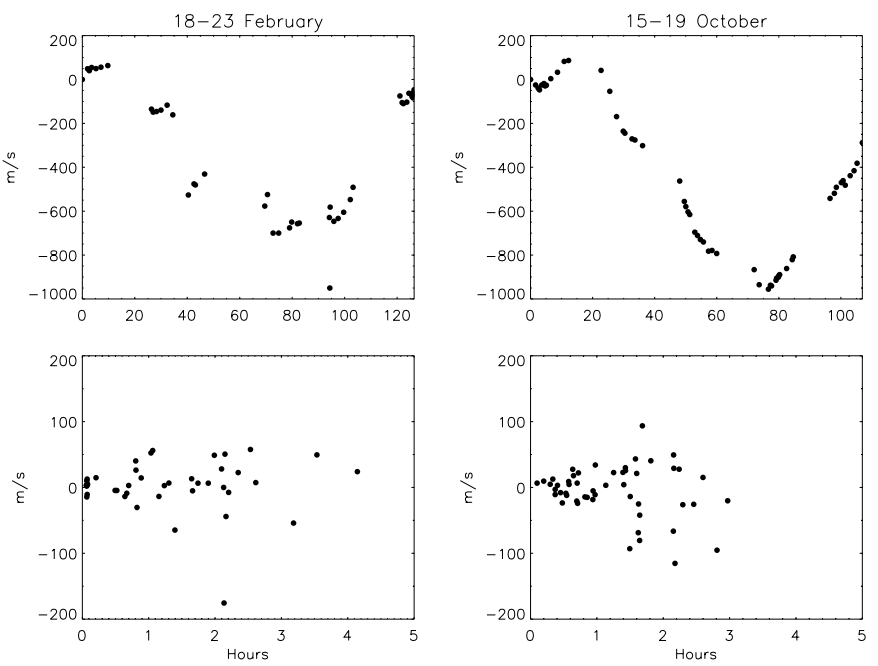

Fig. 3. The left panels show the drift of the Th-Ar calibration for observations made in February 1997; the right panels show the corresponding data for October 1997. The upper panels show the accumulated drift during the nights from an arbitrary origin. The lower panels show the drift between successive $\mathrm{Th}-\mathrm{Ar}$ calibration exposures as function of the time interval between them.
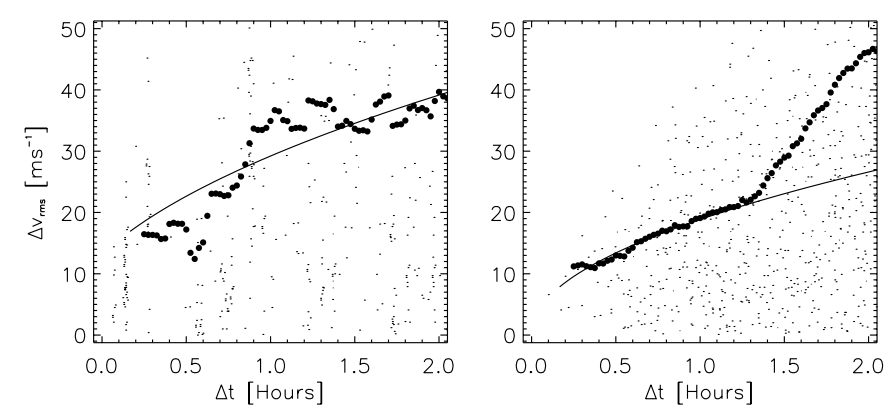

Fig. 4. This plot is similar to the lower panels in Fig. 3, except that more points are added representing all possible data pairs $(\Delta t, \Delta v)$ (i.e. not just successive exposures). The thick dots show a moving average using a window of 0.5 hour. The continuous curves show the fitted function $\Delta v_{\mathrm{rms}}=(a+b \Delta t)^{1 / 2}$ representing a Wiener process plus white noise.

\subsection{Short-term drift correction}

Short-term stability of the ELODIE spectrometer is normally ensured by recording a $\mathrm{Th}-\mathrm{Ar}$ exposure simultaneously with the stellar spectrum. In our case the calibration (Th-Ar) exposures were temporally separated from the stellar or lunar observations, and a small correction for the short-term drift was therefore necessary. The drift in velocity from one calibration exposure to the next is readily derived from the logged data, and allow to reconstruct the drift as function of time from an arbitrary origin (top panels of Fig. 3). Within each night the drift is reasonably smooth, especially in the October data, which makes it meaningful to derive corrections through linear interpolation between successive calibration exposures.

To estimate the uncertainty of such corrections, a statistical model of the drift is needed. The lower panels of Fig. 3 show how the drift $(\Delta v)$ statistically increases with the time interval $(\Delta t)$ between successive exposures. In Fig. 4 the absolute drift values $|\Delta v|$ are shown for all pairs of calibration exposures, together with running averages. We adopt the drift model $E\left(\Delta v^{2}\right)=a+b \Delta t$, i.e. a Wiener (random-walk) process (e.g. Grimmett \& Stirzaker 1982) plus a white-noise term (a) accounting for uncorrelated measurement noise. The fitted curves in Fig. 4 are for

$$
\left.\begin{array}{lll}
a=170 \mathrm{~m}^{2} \mathrm{~s}^{-2}, & b=0.19 \mathrm{~m}^{2} \mathrm{~s}^{-3} & \text { (February) } \\
a=3 \mathrm{~m}^{2} \mathrm{~s}^{-2}, & b=0.10 \mathrm{~m}^{2} \mathrm{~s}^{-3} & \text { (October) }
\end{array}\right\} .
$$

Let $\Delta v_{1}$ (at time $t_{1}$ ) and $\Delta v_{2}$ (at $t_{2}$ ) be two successive drift measurements. The interpolated drift at the intermediate time $t$ is $\Delta v=(1-f) \Delta v_{1}+f \Delta v_{2}$, where $f=\left(t-t_{1}\right) /\left(t_{2}-t_{1}\right)$. (Actually, the correction applied is the drift since the previous Th-Ar calibration exposure, $d_{\mathrm{D}}=\Delta v-\Delta v_{1}$.) The uncertainty of the correction is

$\sigma_{\mathrm{D}}=\sqrt{\left[\frac{1}{2}-f(1-f)\right] a+f(1-f)\left(t_{2}-t_{1}\right) b}$.

A few stellar observations were made after the last Th-Ar exposure of the night, in which case no short-term drift correction was applied. The resulting uncertainty is

$\sigma_{\mathrm{D}}=\sqrt{\frac{1}{2} a+\left(t-t_{1}\right) b}$.

Although the data suggest a much improved instrument stability in the October period, we use the more conservative values $a=170 \mathrm{~m}^{2} \mathrm{~s}^{-2}, b=0.19 \mathrm{~m}^{2} \mathrm{~s}^{-3}$ to estimate the drift uncertainty in both observation periods. With intervals up to $3.5 \mathrm{hr}$ between calibration exposures, the uncertainty of the drift correction could in some cases amount to $25 \mathrm{~m} \mathrm{~s}^{-1}$. The median $\sigma_{\mathrm{D}}$ is $17 \mathrm{~m} \mathrm{~s}^{-1}$.

\subsection{Barycentric correction}

For the observations of stars, the barycentric correction amounts to the application of the two factors in Eq. (1). $v_{\text {LOS }}$ is provided by the ELODIE software for the effective (i.e., flux-weighted) mean time of observation (Baranne et al. 1996). However, in a few cases the timing automatically logged by the ELODIE system was clearly offset, and we therefore chose to re-compute this velocity for all the observations. The mean epoch of observation was reconstructed from the observers' notes, and the barycentric velocity of the observatory $\boldsymbol{v}_{\text {obs }}$ obtained from JPL's Horizons On-Line Ephemeris System (Giorgini et al. 1996; Chamberlin et al. 1997). For the same epoch, the coordinate direction $\boldsymbol{k}$ to the star was computed using data from the Hipparcos Catalogue (Turon et al. 1998). Both vectors are expressed in the ICRF frame, so $v_{\mathrm{LOS}}=\boldsymbol{k}^{\prime} \boldsymbol{v}_{\mathrm{obs}}$ follows as the scalar product. In the mean, the values provided by the ELODIE software agreed well with our calculations, but in 7 cases (out of 76) the difference exceeded $10 \mathrm{~m} \mathrm{~s}^{-1}$, and in 2 cases it exceeded $200 \mathrm{~m} \mathrm{~s}^{-1}$.

For an observation spanning the time interval $\left[t_{\text {beg }}, t_{\text {end }}\right]$ the barycentric correction used $v_{\mathrm{LOS}}\left(t_{\mathrm{mid}}\right)$ computed for the exposure mid-time, $t_{\text {mid }}=\left(t_{\text {beg }}+t_{\text {end }}\right) / 2$. There is an uncertainty in this correction due to the unknown difference between $t_{\text {mid }}$ and the actual flux-weighted mean epoch of observation. We estimate this uncertainty to be around 10 per cent of the 
Table 1. Observations of the Moon, from which the long-term drift correction is determined, and subsequently the barycentric radial-velocity measure of the Sun. The columns contain: Date - mean epoch of observation, given as the topocentric Julian Ephemeris Date (JED) minus $2450000.0 ; d_{\mathrm{N}}(\mathrm{SFA})$ - nominal spectral shift obtained by correlating with the Solar Flux Atlas (Kurucz et al. 1984); $d_{\mathrm{D}}-$ short-term drift correction; $v_{\mathrm{LOS}}$ - line-of-sight velocity (Sun-Moon-observer light path); $d_{\mathrm{B}}$ - resulting barycentric shift with respect to the Solar Flux Atlas; $d_{\mathrm{N}}(\mathrm{Fe} \mathrm{I})-$ nominal shift obtained by correlating with the synthetic Fe I template; $d_{0}-$ long-term drift correction (mean value of $\left.-d_{\mathrm{B}}\right) ; c z_{\mathrm{B}}-$ barycentric radial-velocity measure of the Sun (referring to the Fe I template), including the correction from Eq. (2); mean value and dispersion of the barycentric radial-velocity measures for the 1997 February and October observation periods. Indicated uncertainties are internal standard errors.

\begin{tabular}{ccccccccc}
\hline \hline $\begin{array}{c}\text { Date } \\
2450000+\end{array}$ & $\begin{array}{c}d_{\mathrm{N}}(\mathrm{SFA}) \\
\mathrm{km} \mathrm{s}^{-1}\end{array}$ & $\begin{array}{c}d_{\mathrm{D}} \\
\mathrm{km} \mathrm{s}^{-1}\end{array}$ & $\begin{array}{c}v_{\mathrm{LOS}} \\
\mathrm{km} \mathrm{s}^{-1}\end{array}$ & $\begin{array}{c}d_{\mathrm{B}} \\
\mathrm{km} \mathrm{s}^{-1}\end{array}$ & $\begin{array}{c}d_{\mathrm{N}}(\mathrm{Fe} \mathrm{I}) \\
\mathrm{km} \mathrm{s}^{-1}\end{array}$ & $\begin{array}{c}d_{0} \\
\mathrm{~km} \mathrm{~s}^{-1}\end{array}$ & $\begin{array}{c}c z_{\mathrm{B}} \\
\mathrm{km} \mathrm{s}^{-1}\end{array}$ & $\begin{array}{c}\text { mean value } \\
\mathrm{km} \mathrm{s}^{-1}\end{array}$ \\
\hline 498.2761 & +0.688 & -0.003 & -0.785 & $-0.100 \pm 0.018$ & +0.960 & +0.090 & $+0.267 \pm 0.018$ & \\
498.2819 & +0.704 & -0.007 & -0.794 & $-0.097 \pm 0.021$ & +0.978 & +0.090 & $+0.272 \pm 0.021$ & \\
498.2854 & +0.724 & -0.009 & -0.799 & $-0.084 \pm 0.023$ & +0.999 & +0.090 & $+0.286 \pm 0.023$ & $+0.277 \pm 0.010$ (Feb.) \\
498.2899 & +0.720 & -0.011 & -0.806 & $-0.097 \pm 0.024$ & +0.992 & +0.090 & $+0.270 \pm 0.024$ & \\
498.2927 & +0.749 & -0.013 & -0.810 & $-0.074 \pm 0.026$ & +1.021 & +0.090 & $+0.293 \pm 0.026$ & \\
737.3799 & -0.537 & -0.002 & +0.556 & $+0.017 \pm 0.011$ & -0.302 & -0.016 & $+0.240 \pm 0.011$ & \\
737.3841 & -0.518 & -0.002 & +0.550 & $+0.030 \pm 0.013$ & -0.284 & -0.016 & $+0.252 \pm 0.013$ & \\
737.3910 & -0.520 & -0.002 & +0.539 & $+0.017 \pm 0.009$ & -0.286 & -0.016 & $+0.240 \pm 0.009$ & \\
740.4709 & -1.152 & -0.001 & +1.163 & $+0.010 \pm 0.010$ & -0.921 & -0.016 & $+0.229 \pm 0.010$ & $+0.238 \pm 0.008$ (Oct.) \\
740.4750 & -1.151 & -0.001 & +1.158 & $+0.006 \pm 0.011$ & -0.917 & -0.016 & $+0.228 \pm 0.011$ & \\
740.4778 & -1.133 & -0.002 & +1.154 & $+0.019 \pm 0.013$ & -0.897 & -0.016 & $+0.243 \pm 0.013$ & \\
740.4806 & -1.133 & -0.003 & +1.150 & $+0.014 \pm 0.013$ & -0.902 & -0.016 & $+0.233 \pm 0.013$ & \\
\hline
\end{tabular}

variation of the barycentric correction over the exposure, or $\sigma_{\text {LOS }}=0.1 \times\left|v_{\text {LOS }}\left(t_{\text {end }}\right)-v_{\text {LOS }}\left(t_{\text {beg }}\right)\right|$. The median uncertainty per observation from this effect is $13 \mathrm{~m} \mathrm{~s}^{-1}$.

The observations of the Moon, in the upper branch of Fig. 1, receive a corresponding barycentric correction, including the factor Eq. (2), only with $v_{\mathrm{LOS}}$ computed through numerical differentiation of the total path length from the Sun to the observer, $\left|\boldsymbol{r}_{\mathrm{M}}\left(t_{\mathrm{M}}\right)-\boldsymbol{r}_{\odot}\left(t_{\odot}\right)\right|+\left|\boldsymbol{r}_{\mathrm{M}}\left(t_{\mathrm{M}}\right)\right|$. Here $\boldsymbol{r}_{\odot}(t)$ and $\boldsymbol{r}_{\mathrm{M}}(t)$ are the geometric ephemerides of the Sun and the subterrestrial point on the Moon, respectively, relative the observer; $t_{\mathrm{M}}$ and $t_{\odot}$ are the time of observation diminished by the light time to the respective object. Relative geometric coordinates were obtained via the JPL Horizons system. In this calculation it was assumed that the telescope was pointed at the geometrical centre of the lunar disk. This is not a critical issue: a depointing by one tenth of the moon's apparent diameter would at most cause an error of $0.6 \mathrm{~m} \mathrm{~s}^{-1}$ in the barycentric correction.

In the lower branch of Fig. 1 the observations are correlated with the Solar Flux Atlas (Kurucz et al. 1984), and the barycentric correction must here be defined as was done for the Atlas. From the description of the latter we infer that no correction corresponding to Eq. (2) was used in constructing its rest wavelength scale. Consequently, in the lower branch of Fig. 1, the barycentric correction amounts only to the factor $1+v_{\mathrm{LOS}} / c$.

\subsection{Long-term drift correction (absolute zero point)}

The long-term drift correction is computed on the assumption that the solar spectrum has no intrinsic long-term velocity variations and that the wavelength scale in the Solar Flux Atlas is correct. These assumptions are further discussed in Sect. 7.1.

The correlation of a Moon spectrum with the Solar Flux Atlas gives the shift $d_{\mathrm{N}}$ (SFA) in the second column of Table 1 . This is expressed on the nominal wavelength scale of the previous Th-Ar exposure. After correction for the short-term drift $\left(d_{\mathrm{D}}\right)$ and line-of-sight velocity $\left(v_{\mathrm{LOS}}\right)$ we obtain the barycentric quantity $d_{\mathrm{B}}$, which should be zero if the Th-Ar wavelengths are effectively on the same scale as the wavelengths in the Solar Flux Atlas. As shown in the table, $d_{\mathrm{B}}$ is significantly different between the February and October sessions (while the variations within each session are hardly significant). As discussed in Sect. 7.1, it is likely that this difference is (mainly) an instrumental effect, perhaps resulting from some readjustment of the spectrometer made between the two observing sessions.

Accordingly, we adopt the mean $-d_{\mathrm{B}}$ in each observing period as the long-term drift correction, or absolute zero point $\left(d_{0}\right)$ for the radial-velocity measures. This gives $d_{0}=$ $+0.090 \pm 0.010 \mathrm{~km} \mathrm{~s}^{-1}$ for the February data, and $d_{0}=-0.016 \pm$ $0.007 \mathrm{~km} \mathrm{~s}^{-1}$ for October. For both periods we adopt $\sigma_{0}=$ $0.010 \mathrm{~km} \mathrm{~s}^{-1}$ as the zero-point uncertainty.

\subsection{Combined corrections}

The observed spectral shift, corrected for short-term drift and zero point, is given by

$c \ln \left(1+z_{\mathrm{obs}}\right)=d_{\mathrm{N}}(\mathrm{Fe} \mathrm{I})+d_{\mathrm{D}}+d_{0}$.

Inserting this in Eq. (1) and expanding exponentials to second order gives

$c z_{\mathrm{B}}=D+\frac{1}{2 c}\left(D^{2}-v_{\mathrm{LOS}}^{2}\right)$,

where

$D=d_{\mathrm{N}}(\mathrm{Fe} \mathrm{I})+d_{\mathrm{D}}+v_{\mathrm{LOS}}+d_{0}+\left(4.44 \mathrm{~m} \mathrm{~s}^{-1}\right)$

(this approximation should not be used for velocities above some $600 \mathrm{~km} \mathrm{~s}^{-1}$, cf. Sect. 3). 


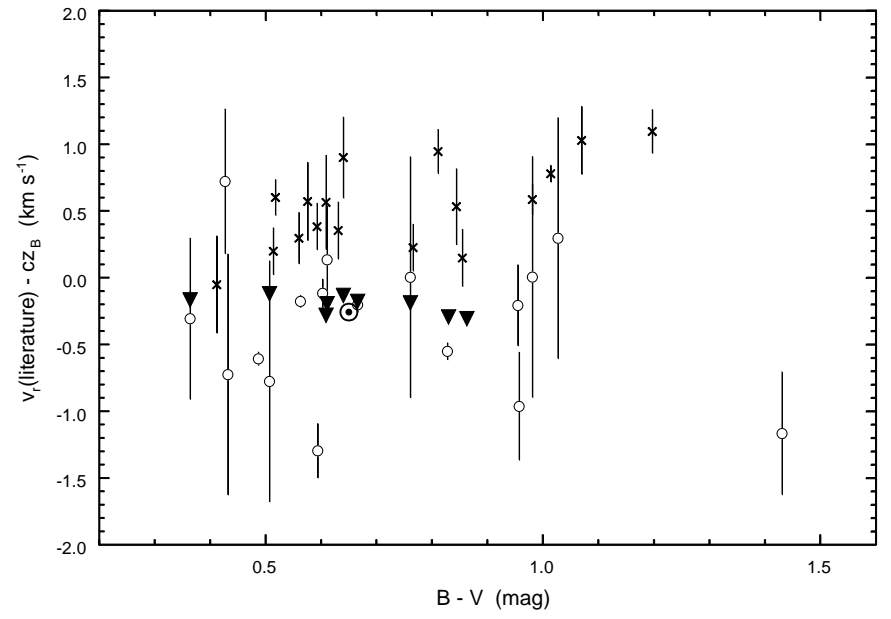

Fig. 5. Differences between various radial-velocity determinations in the literature $\left(v_{\mathrm{r}}(\mathrm{CDS})\right.$ or $v_{\mathrm{r}}$ (other) in Table 2$)$ and our radial-velocity measures, plotted against colour index. Error bars show $\pm 1 \sigma$, where $\sigma$ is the sum in quadrature of the uncertainties in Table 2. Only data with $\sigma<1 \mathrm{~km} \mathrm{~s}^{-1}$ are plotted. Crosses are for $v_{\mathrm{r}}$ by Griffin et al. (1988), filled triangles by Nidever et al. (2002), and open circles from other sources. For the Nidever et al. differences, the error bars are too small to be visible in the diagram. The solar symbol shows the difference for the Sun, knowing its $v_{\mathrm{r}}=0$.

The total internal error of $c z_{\mathrm{B}}$ is obtained as the sum in quadrature of the standard errors of the terms in Eq. (11), viz. $\sigma_{\mathrm{N}}=c \sigma_{\hat{u}}$ from Eq. (A.3), $\sigma_{\mathrm{D}}$ from Eq. (7) or (8), $\sigma_{\mathrm{LOS}}$ from Sect. 5.4, and $\sigma_{0}$ from Sect. 5.5. The error in the last term of Eq. (11) is neglected. In the cases where the final radialvelocity measure is computed as a mean of $N>1$ observations, $\sigma_{0}$ is applied after the averaging (thus $\sigma_{0}$ is not reduced by $N^{-1 / 2}$ ). Typical values of the errors are summarised in Table 3.

\section{Results}

Resulting barycentric radial-velocity measures for the Sun (lunar spectra) are given in the rightmost columns of Table 1, and for the stars in Table 2. The indicated uncertainties are internal standard errors. The lunar observations show an apparent change by $39 \mathrm{~m} \mathrm{~s}^{-1}$ between February and October. We believe this is related to the instrumental changes discussed in Sect. 5.5 and therefore indicative of a minimum level of external errors in these results as well as for the stars. The unweighted mean from the two periods gives $c z_{\mathrm{B}}=+0.257 \mathrm{~km} \mathrm{~s}^{-1}$ as our best estimate of the full-disk solar radial-velocity measure (its standard error, including the zero-point uncertainty $\sigma_{0}$, is $0.011 \mathrm{~m} \mathrm{~s}^{-1}$ ). This is consistent with typical shifts of mediumstrong Fe I lines (equivalent width $\simeq 6 \mathrm{pm}$ ) as measured in the Solar Flux Atlas by Allende Prieto \& García López (1998b), although their data refer to the line bottoms while our results are for points higher up towards the continuum (cf. Sect. 7.2). Subtracting the gravitational redshift for an observer at infinity $\left(636.5 \mathrm{~m} \mathrm{~s}^{-1}\right)$ gives a net blueshift of $-379 \mathrm{~m} \mathrm{~s}^{-1}$ relative to the $\mathrm{Fe}$ I template due to convection and possibly other effects.

When more than one good spectrum was obtained of the same star in the same observation period, Table 2 gives the weighted mean radial-velocity measure at the similarly weighted mean observation epoch; the internal standard error of the mean was calculated from the total statistical weight. The chi-square of the residuals with respect to the mean value was acceptable in all except two of these cases. For HIP 71284 (28 Boo, a known variable star) the two observations separated by only $40 \mathrm{~min}$ were marginally discordant $\left(c z_{\mathrm{B}}=\right.$ $+0.472 \pm 0.091$ and $+0.225 \pm 0.064 \mathrm{~km} \mathrm{~s}^{-1}$ ). The other case is HIP 113357, the well-known exoplanet system 51 Peg (Mayor \& Queloz 1995), where the four measurements gave $\chi^{2}=32$ (3 degrees of freedom). This was reduced to a satisfactory $\chi^{2}=2.5$ (3 degrees of freedom) after correction for the planetinduced velocity using the orbital period and phase from Bundy \& Marcy (2000), whose observations span our data, and the velocity amplitude from Marcy et al. (1997). The mean value in Table 2 is for the corrected values and thus refers to the centre of gravity of the system.

Three stars (besides the Sun) were observed in both observation periods, for which (mean) results are given on separate lines in Table 2. For two of them (HIP 20205 and 87079) there is excellent agreement between the two measures. For the Hyades K giant HIP20889 (= $\epsilon$ Tau $=$ vB 70) the two measures differ by $0.1 \mathrm{~km} \mathrm{~s}^{-1}$, significant at the $5 \sigma$ level. This star was separately discussed by Griffin et al. (1988), who remarked upon its possible variability on the level of a few tenths of a $\mathrm{km} \mathrm{s}^{-1}$.

Table 2 also gives radial velocities from various other sources. The column $v_{\mathrm{r}}(\mathrm{CDS})$ contains values from the SIMBAD data base (Centre de Données astronomiques de Strasbourg), while $v_{\mathrm{r}}$ (other) contains more precise values from the literature when available. A comparison of these data with our radial-velocity measures is shown in Fig. 5. The location of the Sun in the diagram is shown by the solar symbol at $B-V=0.65, v_{\mathrm{r}}-c z_{\mathrm{B}}=-0.257 \mathrm{~km} \mathrm{~s}^{-1}$. Two sets of differences are especially worth noting. (1) The crosses are for the photoelectric radial velocities of Hyades stars by Griffin et al. (1988). The mean difference for the stars with $B-V<0.9$ (which excludes the two giants) is $+0.45 \mathrm{~km} \mathrm{~s}^{-1}$, with some apparent trend depending on colour index or magnitude. Compared with the solar value, this suggests that the Griffin et al. velocities require a correction of $\simeq-0.7 \mathrm{~km} \mathrm{~s}^{-1}$ for the late $\mathrm{F}$ and $\mathrm{G}$ stars in order to put them on the kinematic velocity scale. (2) The filled triangles are for the nine stars in common with the list of very precise absolute radial velocities by Nidever et al. (2002). Here, the mean difference is $-0.21 \mathrm{~km} \mathrm{~s}^{-1}$ and the RMS scatter of differences only $0.07 \mathrm{~km} \mathrm{~s}^{-1}$. Nidever et al. use the Solar Flux Atlas as a template for the F, G and K stars, so the mean difference is expected to be close to the solar value $-0.257 \mathrm{~km} \mathrm{~s}^{-1}$. Thus their velocity scale is consistent with ours to within $0.05 \mathrm{~km} \mathrm{~s}^{-1}$. The differences based on radial velocities from various other sources agree on the average with the Nidever et al. data, although the scatter is substantial.

Ultimately the present radial-velocity measures may be compared with astrometric radial velocities, such as those obtained by Madsen et al. (2002), in order to derive the spectral line shifts caused by convection and other intrinsic stellar effects. However, such a comparison requires detailed consideration of many additional factors, and is therefore beyond the scope of this paper. 
Table 2. Results of the stellar observations, given in the form of barycentric radial-velocity measures $c z_{\mathrm{B}}$ (referring to the synthetic Fe $\mathrm{I}$ template), and a comparison with published radial velocities $v_{\mathrm{r}}$. Note that for HIP 20205, 20889, 87079 there are two lines of data per star, one for each observing period. The columns contain: HIP - Hipparcos Catalogue number; HD/HDE/BD - alternative designation; Sp - spectral type; $B-V-$ colour index (Sp and $B-V$ from Hipparcos Catalogue Turon et al. 1998); Date - mean epoch of observation, given as the barycentric Julian Ephemeris Date (JED) minus 2450000.0; $c z_{\mathrm{B}}$ - (mean) radial-velocity measure and estimated internal standard error; $N$ number of observations; $v_{\mathrm{r}}(\mathrm{CDS})$ and $v_{\mathrm{r}}$ (other) - radial velocity from the SIMBAD data base (CDS, Strasbourg) and other sources; Ref. reference for radial velocity (see below); Rem. - remark concerning cluster membership (Hyades or Ursa Major), radial-velocity standard star (std), metal-poor star (mp), or other. For Hyades stars in Griffin et al. (1988) we also give the designation used in their Table IV. See text for comments on the (variable) stars HIP 71284 and 113357.

\begin{tabular}{|c|c|c|c|c|c|c|c|c|c|c|c|}
\hline HIP & $\begin{array}{r}\mathrm{HD} / \mathrm{HDE} \\
\mathrm{BD}\end{array}$ & $\mathrm{Sp}$ & $\begin{array}{c}B-V \\
\text { mag }\end{array}$ & $\begin{array}{c}\text { Date } \\
2450000+\end{array}$ & $\begin{array}{c}c z_{\mathrm{B}} \\
\mathrm{km} \mathrm{s}^{-1}\end{array}$ & $N$ & $\begin{array}{r}v_{\mathrm{r}}(\mathrm{CDS}) \\
\mathrm{km} \mathrm{s}^{-1}\end{array}$ & Ref. & $\begin{array}{c}v_{\mathrm{r}} \text { (other) } \\
\mathrm{km} \mathrm{s}^{-1}\end{array}$ & Ref. & Rem. \\
\hline 910 & 693 & $\mathrm{~F} 5 \mathrm{~V}$ & 0.487 & 741.4221 & $+15.107 \pm 0.027$ & 1 & $+14.4 \pm 0.9$ & 2 & $+14.50 \pm 0.04$ & 6 & std \\
\hline 13806 & +29503 & G5 & 0.855 & 740.6419 & $+26.472 \pm 0.027$ & 1 & $+22.7 \pm 2.0$ & 1 & $+26.62 \pm 0.21$ & 3 & Hya (vB 153) \\
\hline 13834 & 18404 & F5IV & 0.415 & 501.2739 & $+27.974 \pm 0.106$ & 1 & $+28.1 \pm 2.0$ & 2 & & & Hya \\
\hline 15720 & - & - & 1.431 & 739.5265 & $+30.066 \pm 0.085$ & 1 & & & $+28.90 \pm 0.45$ & 5 & Hya \\
\hline 19504 & 26345 & F6V & 0.427 & 737.4273 & $+36.379 \pm 0.449$ & 1 & $+34.5 \pm 2.0$ & 2 & $+37.10 \pm 0.30$ & 5 & Hya (vB 13) \\
\hline 19655 & - & MOV: & 1.216 & 739.5412 & $-10.156 \pm 0.022$ & 2 & & & & & UMa \\
\hline 19786 & 26767 & G0 & 0.640 & 741.5809 & $+38.420 \pm 0.027$ & 1 & $+39.1 \pm 2.0$ & 1 & $+38.29 \pm 0.03 \dagger$ & 9 & Hya (vB 18) \\
\hline 19796 & 26784 & F8V & 0.514 & 738.4939 & $+38.302 \pm 0.089$ & 1 & $+36.8 \pm 2.0$ & 2 & $+38.50 \pm 0.15$ & 3 & Hya (vB 19) \\
\hline 20205 & 27371 & G8III & 0.981 & 498.3456 & $+38.696 \pm 0.014$ & 2 & $+38.7 \pm 0.9$ & 2 & $+39.28 \pm 0.11$ & 3 & Hya (vB 28) \\
\hline 20889 & 28305 & KOIII & 1.014 & 498.3724 & $+38.641 \pm 0.013$ & 2 & $+39.0 \pm 5.0$ & 2 & $+39.37 \pm 0.06$ & 3 & Hya (vB 70) \\
\hline 20889 & 28305 & KOIII & 1.014 & 745.5351 & $+38.541 \pm 0.013$ & 1 & $+39.0 \pm 5.0$ & 2 & $+39.37 \pm 0.06$ & 3 & Hya (vB 70) \\
\hline 20899 & 28344 & $\mathrm{G} 2 \mathrm{~V}$ & 0.609 & 501.3387 & $+39.426 \pm 0.027$ & 1 & $+39.8 \pm 2.0$ & 2 & $+39.15 \pm 0.03 \neq$ & 9 & Hya (vB 73) \\
\hline 20949 & 283704 & G5 & 0.766 & 737.5852 & $+38.795 \pm 0.030$ & 1 & $+36.8 \pm 2.0$ & 1 & $+39.02 \pm 0.17$ & 3 & Hya (vB 76) \\
\hline 21261 & 285837 & - & 1.197 & 745.6615 & $+40.335 \pm 0.058$ & 1 & & & $+41.43 \pm 0.15$ & 3 & Hya (J 291) \\
\hline 21317 & 28992 & G1V & 0.631 & 737.4956 & $+40.427 \pm 0.026$ & 1 & $+42.0 \pm 5.0$ & 1 & $+40.78 \pm 0.21$ & 3 & Hya (vB 97) \\
\hline 21637 & 29419 & F5 & 0.576 & 502.3667 & $+39.289 \pm 0.023$ & 1 & $+40.0 \pm 5.0$ & 1 & $+39.86 \pm 0.29$ & 3 & Hya (vB 105) \\
\hline 21741 & 284574 & $\mathrm{~K} 0 \mathrm{~V}$ & 0.811 & 738.6277 & $+40.395 \pm 0.032$ & 1 & $+39.5 \pm 2.0$ & 1 & $+41.34 \pm 0.16$ & 3 & Hya (vB 109) \\
\hline 22654 & 284930 & K0 & 1.070 & 745.5827 & $+41.852 \pm 0.033$ & 1 & & & $+42.88 \pm 0.25$ & 3 & Hya (L 98) \\
\hline 23214 & 31845 & F5V & 0.450 & 739.5863 & $+42.561 \pm 0.436$ & 1 & $+44.1 \pm 2.0$ & 1 & $+42.50 \pm 1.50$ & 5 & Hya \\
\hline 23312 & +04810 & $\mathrm{~K} 2$ & 0.957 & 739.6266 & $+43.173 \pm 0.037$ & 1 & & & $+42.21 \pm 0.40$ & 5 & Hya \\
\hline 84195 & 155712 & K0 & 0.941 & 738.2800 & $+19.991 \pm 0.027$ & 1 & & & & & UMa \\
\hline 87079 & 163183 & G0 & 0.619 & 499.6231 & $-4.544 \pm 0.024$ & 2 & & & & & UMa \\
\hline 87079 & 163183 & G0 & 0.619 & 738.7318 & $-4.572 \pm 0.025$ & 6 & & & & & UMa \\
\hline 95447 & 182572 & G8IVvar & 0.761 & 740.3722 & $-100.104 \pm 0.017$ & 1 & $-100.1 \pm 0.9$ & 2 & $-100.29 \pm 0.03$ & 9 & std \\
\hline 97675 & 187691 & F8V & 0.563 & 738.4285 & $+0.217 \pm 0.031$ & 1 & $-0.2 \pm 0.9$ & 2 & $+0.04 \pm 0.03$ & 6 & std \\
\hline 102040 & 197076 & G5V & 0.611 & 745.3125 & $-35.215 \pm 0.021$ & 1 & $-37.0 \pm 2.0$ & 1 & $-35.41 \pm 0.03$ & 9 & std \\
\hline 106278 & 204867 & $\mathrm{G} 0 \mathrm{Ib}$ & 0.828 & 737.3641 & $+6.911 \pm 0.013$ & 2 & $+6.5 \pm 0.9$ & 1 & $+6.36 \pm 0.06$ & 6 & std \\
\hline 113357 & 217014 & G5V & 0.666 & 739.5116 & $-33.048 \pm 0.012$ & 4 & $-31.2 \pm 2.0$ & 1 & $-33.23 \pm 0.03$ & 9 & 51 Peg (var) \\
\hline 115949 & 221170 & G2IV & 1.027 & 739.3436 & $-121.696 \pm 0.027$ & 1 & $-119.0 \pm 5.0$ & 1 & $-121.40 \pm 0.90$ & 7 & $\mathrm{mp}$ \\
\hline 116771 & 222368 & F7V & 0.507 & 739.3876 & $+5.776 \pm 0.016$ & 3 & $+5.0 \pm 0.9$ & 1 & $+5.66 \pm 0.03$ & 9 & std \\
\hline
\end{tabular}

$\dagger$ Griffin et al. (1988) give $v_{\mathrm{r}}=+39.32 \pm 0.30 . \quad \ddagger$ Griffin et al. $(1988)$ give $v_{\mathrm{r}}=+39.99 \pm 0.35$.

References: 1. GCSRV (Wilson 1953); 2. Revised GCRV (Evans 1967); 3. Griffin et al. (1988, quoted error is the larger of the internal and external errors); 4. Soderblom \& Mayor (1993); 5. Perryman et al. (1998); 6. Stefanik et al. (1999); 7. Barbier-Brossat \& Figon (2000); 8. Beers et al. (2000); 9. Nidever et al. (2002).

\section{Discussion of the method}

\subsection{The absolute zero point}

The absolute zero point of the radial-velocity measures, which is effectively set by the long-term drift corrections described in
Sect. 5.5, rests on the assumptions (1) that the integrated solar disk spectrum has no significant long-term velocity variation; (2) that the ELODIE instrument has sufficient long-term stability; (3) that the wavelength scale in the Solar Flux Atlas correctly represents the measurements of an observer in circular 
Table 3. Typical (median) random errors of the various reduction steps contributing to the final standard errors of the radial-velocity measures in Table 2, and estimated systematic errors. A cautionary note: while the random errors are well quantified using theory (e.g. based on photon statistics) or the results of repeated measurements, the systematic errors are little more than order-of-magnitude estimates as discussed in Sect. 7.1.

\begin{tabular}{lr}
\hline \hline Random errors (median values): & \\
- cross-correlation $\left(\sigma_{\mathrm{N}}\right)$, Appendix A & $13 \mathrm{~m} \mathrm{~s}^{-1}$ \\
- short-term drift $\left(\sigma_{\mathrm{D}}\right)$, Eqs. (7)-(8) & $17 \mathrm{~m} \mathrm{~s}^{-1}$ \\
- barycentric correction $\left(\sigma_{\mathrm{LOS}}\right)$, Sect. 5.4 & $13 \mathrm{~m} \mathrm{~s}^{-1}$ \\
- long-term drift $\left(\sigma_{0}\right)$, Sect. 5.5 & $10 \mathrm{~m} \mathrm{~s}^{-1}$ \\
total median standard error & $27 \mathrm{~m} \mathrm{~s}^{-1}$ \\
& \\
Estimated systematic errors: & $30 \mathrm{~m} \mathrm{~s}^{-1}$ \\
- long-term instability of the solar spectrum & $50 \mathrm{~m} \mathrm{~s}^{-1}$ \\
- long-term instability of the instrument & $100 \mathrm{~m} \mathrm{~s}^{-1}$ \\
- wavelength scale of the Solar Flux Atlas & $30 \mathrm{~m} \mathrm{~s}^{-1}$ \\
- laboratory Fe I wavelengths & $120 \mathrm{~m} \mathrm{~s}^{-1}$ \\
total systematic error
\end{tabular}

orbit at $1 \mathrm{AU}$ distance from the Sun; and (4) that the laboratory wavelengths, in this case for the Fe I lines, have no zero point error. Below, we discuss each of these assumptions.

The long-term wavelength stability of line shifts and asymmetries in the solar spectrum has been the subject of several investigations. Concerning line shifts, McMillan et al. (1993) found an upper limit of $\pm 4 \mathrm{~m} \mathrm{~s}^{-1}$ for the variation of ultraviolet absorption lines over a solar cycle; while Deming \& Plymate (1994) found an amplitude of $10-15 \mathrm{~m} \mathrm{~s}^{-1}$ in the infrared. The continuous low-frequency velocity spectrum was measured e.g. by Pallé et al. (1995), who found a mean spectral density $<3 \times 10^{5} \mathrm{~m}^{2} \mathrm{~s}^{-2} \mathrm{~Hz}^{-1}$ for frequencies below $10^{-5} \mathrm{~Hz}$, i.e. on time scales longer than $\sim 1$ day. For daily averages, this implies an amplitude less than a few $\mathrm{m} \mathrm{s}^{-1}$. Livingston et al. (1999) followed the full-disk asymmetries of Fe I lines for more than a solar cycle and found cyclic variations in the line asymmetry with an amplitude of about $20 \mathrm{~m} \mathrm{~s}^{-1}$; presumably the corresponding absolute shifts are at least of a similar size. Variations of the order 20-30 $\mathrm{m} \mathrm{s}^{-1}$ are also predicted from spatially resolved observations in active regions (e.g. Brandt \& Solanki 1990; Spruit et al. 1990) combined with the known long-term variations in their fractional coverage of the solar disk (e.g. Tang et al. 1984). For a general discussion of solar-cycle variations, see also Dravins (1999).

Thus, although direct observations are inconclusive, there are good reasons to expect long-term variations of the visual solar line shifts of the order $30 \mathrm{~m} \mathrm{~s}^{-1}$. The results of Livingston et al. (1999) suggest that such changes may occur on time scales of a year or less. We cannot therefore rule out that the solar spectrum changed significantly between February and October 1997, although instrumental effects remain a more likely explanation for the systematic difference of $\simeq 100 \mathrm{~m} \mathrm{~s}^{-1}$ between the two observation sessions, as assumed in Sect. 5.5.

In a general sense, the ELODIE instrument has a proven long-term stability, which may be better than $10 \mathrm{~m} \mathrm{~s}^{-1}$
(Udry et al. 1999). However, the instrument was here used in a non-standard mode with time-separated Th-Ar exposures and with the Moon spectrum as an intermediate reference. The use of an extended source like the Moon for wavelength calibration is traditionally frown upon (see, e.g., Sect. VIIb in Griffin et al. 1988), but the use of fibre-fed échelle spectrometers has probably eliminated much of that problem (Baranne 1999). Even so, the systematic difference of $39 \mathrm{~m} \mathrm{~s}^{-1}$ between the February and October results for the Moon (Table 1) indicates some additional effect in our data. The behaviour of $d_{\mathrm{B}}$ versus the FWHM of the synthetic template is very different in the two observation sessions, which leads us to believe that the systematic difference could be explained by a slight change in the asymmetry of the instrument profile, perhaps due to some readjustment of the spectrometer in the intervening period. In view of such results we estimate that the instrumental contributions to the standard error of the zero point are of the order $50 \mathrm{~m} \mathrm{~s}^{-1}$.

The Solar Flux Atlas from 296 to $1300 \mathrm{~nm}$ by Kurucz et al. (1984) is a spectrum of the disk-integrated sunlight obtained with a FTS yielding a spectral resolution ranging from 348000 in ultraviolet to 522000 in red and infrared, and with $S / N$ up to 9000. The wavelength scale has been corrected for Sun-Earth velocity shifts, but the gravitational redshift relative to a terrestrial observer $\left(633.5 \mathrm{~m} \mathrm{~s}^{-1}\right)$ was not removed, nor, presumably, the transverse Doppler shift from the Earth's orbital motion $\left(1.5 \mathrm{~m} \mathrm{~s}^{-1}\right)$. Each scan of the FTS covering a certain spectral region provided an intrinsically uniform wavelength scale, but a multiplicative factor had to be determined by means of the telluric $\mathrm{O}_{2}$ line at $688.38335 \mathrm{~nm}$. As this was only observed in some scans, the resulting scale was transferred to the other scans by matching overlapping parts of the spectra. As a result of this fitting and shifting, Kurucz et al. consider that the final wavelengths may have errors up to $100 \mathrm{~m} \mathrm{~s}^{-1}$, especially in the ultraviolet end. Allende Prieto \& García López (1998b) examined the absolute wavelength calibration of the Solar Flux Atlas by computing the shifts for the minima of $1446 \mathrm{Fe}$ I lines. They found that lines with equivalent widths $>20$ pm have a mean shift within $20 \mathrm{~m} \mathrm{~s}^{-1}$ of the value expected from non-kinematic effects $\left(632.0 \mathrm{~m} \mathrm{~s}^{-1}\right)$, with a scatter of $58 \mathrm{~m} \mathrm{~s}^{-1}$. Moreover, there is no visible trend with wavelength. From this we conclude that the wavelength scale of the Atlas is probably at least as good as claimed by its authors, i.e. with a zero point error less than $100 \mathrm{~m} \mathrm{~s}^{-1}$. Thanks to the expected wavelength coherency within each scan of the Atlas it may be possible to improve this zero point a posteriori.

Concerning the accuracy of the laboratory wavelengths, Nave et al. (1994) quote systematic errors of the order $0.001 \mathrm{~cm}^{-1}$ due to the calibration error for each laboratory spectrum, and possible pressure or current-dependent shifts estimated to be less than $0.001 \mathrm{~cm}^{-1}\left(=15 \mathrm{~m} \mathrm{~s}^{-1}\right.$ at $\left.\lambda=500 \mathrm{~nm}\right)$ for lines with upper levels of low excitation $(<6 \mathrm{eV})$. Since a majority of the lines used to construct our Fe I template have excitation levels below $6.5 \mathrm{eV}$ we estimate that the total systematic error due to these factors is less than $30 \mathrm{~m} \mathrm{~s}^{-1}$.

Combining the various estimated contributions to the zero point error (Table 3), we find that the total uncertainty is of the order $120 \mathrm{~m} \mathrm{~s}^{-1}$. The main uncertainty comes from the wavelength scale of the Solar Flux Atlas. If that could be improved, 


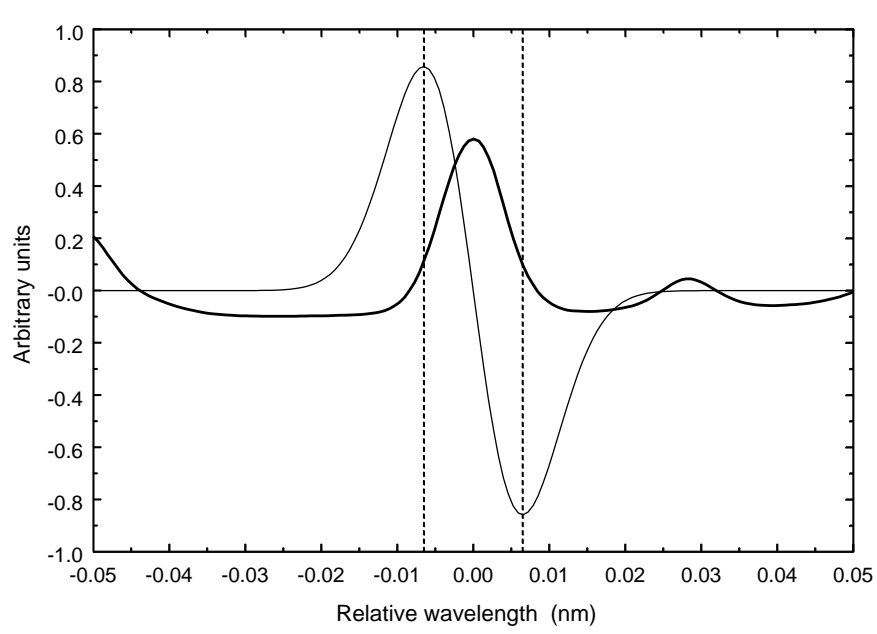

Fig. 6. Illustration of the effective weighting function for the crosscorrelation method used in this paper. The thick curve is an inverted portion of the disk-integrated solar spectrum (Kurucz et al. 1984) centred on the $\mathrm{Fe}_{\mathrm{I}}$ line at $522.55327 \mathrm{~nm}$. The weighting function, shown by the thin curve, is the derivative of a Gaussian with $F W H M=8.8 \mathrm{~km} \mathrm{~s}^{-1}$. The centre of the spectral line is determined by nulling the cross-correlation of the two functions. The weighting function thus defines which parts of the spectral line are used to derive its shift.

as suggested above, it would immediately result in much more accurate radial-velocity measures for all the stars in Table 2, via a simple zero-point correction.

\subsection{Which spectral features are measured?}

The radial-velocity measure refers to specific features in the observed stellar spectrum, and may well be different for different atomic species, or depending on which parts of the spectral lines are used. When publishing radial-velocity measures purporting to be accurate at the $100 \mathrm{~m} \mathrm{~s}^{-1}$ level, it is necessary to specify, as well as possible, to which spectral features they refer. The selection of spectral lines is in our case given by the list of $\mathrm{Fe}_{\mathrm{I}}$ lines used to build the synthetic template. This list is available on request from the authors.

Given that all stellar absorption lines are in reality asymmetric, the way to determine their centres is also of primary concern. The line centres are implicitly defined by the cross-correlation method in combination with the instrument. To see how this works, consider that the true stellar spectrum is smeared first by the instrumental profile (having a $F W H M$ of $\simeq 7.2 \mathrm{~km} \mathrm{~s}^{-1}$; Baranne et al. 1996) and then, in the cross-correlation, by the template profile (which in our case is Gaussian with $F W H M=5 \mathrm{~km} \mathrm{~s}^{-1}$ ). The combined profile $(p)$ is approximately Gaussian with $F W H M=\left(7.2^{2}+5^{2}\right)^{1 / 2} \simeq$ $8.8 \mathrm{~km} \mathrm{~s}^{-1}$. It is mainly this combined profile that defines the centroid position with respect to the true stellar spectrum. In maximising the CCF, the relative weights assigned to the different parts of a spectral line are given by the derivative $p^{\prime}$ (Fig. 6). This "weighting function" (Lindegren 1978) has its extreme points at $\pm 3.7 \mathrm{~km} \mathrm{~s}^{-1}$ (at the dotted lines in the figure), and it is roughly these two points in the absorption line that are balanced against each other. Depending on the actual width of the stellar line, this may happen deep in the line (e.g. for a rotationally broadened spectrum) or nearer the continuum (for a sharp-lined spectrum).

\subsection{Accuracy versus precision}

Our procedure is intended to yield radial-velocity measures that are accurate (or absolute) in the sense that successive improvements of the technique should yield results that approach the true values. In practice it means that the values should be reproducible with other instruments and procedures having similar aims. Thus, as discussed above, special care was taken to ensure that the results refer to an absolute wavelength scale and to known and well-defined features in the stellar spectra, through procedures that might be repeated on another instrument.

Much effort in recent radial-velocity work has focused on achieving the very high precisions required to search for exoplanets. Such techniques, which aim at detecting small changes in the velocity but where the zero point is of little interest, could properly be called accelerometry (Connes 1985). The very high stability of instruments such as ELODIE is a direct consequence of such efforts. While our results benefit much from this stability, our goal is rather different from accelerometry, and many of the techniques developed for that purpose are not applicable here.

In fact, to achieve high accuracy usually means that some precision must be sacrificed. This may at first seem paradoxical, since accuracy implies precision (but not vice versa). However, it is a practical consequence of the limited signalto-noise ratio in available data. Two circumstances can serve to illustrate this.

Firstly, to derive absolute line shifts obviously requires that only spectral lines with accurate laboratory wavelengths can be used. At accuracy levels below $100 \mathrm{~m} \mathrm{~s}^{-1}$ this very severely limits the number of available lines. This would not be a problem if each line could be measured with infinite signal-to-noise ratio, but in a real situation it means that precision is reduced compared to using all measurable lines in the stellar spectrum.

Secondly, as pointed out by Butler et al. (1996), a truly photon-noise limited Doppler analysis must consist of a full model of the spectroscopic observation. This can be achieved by fitting a model spectrum, affected by all the instrumental effects, to the observed data, using the appropriate statistical weighting of each pixel. In practice, the most precise model spectrum available for any given star is simply the mean observed spectrum of that star, recorded with the same instrument setup. This would allow to measure the relative shifts of the individual spectra with optimal precision, but not at all accurate.

Thus, a proper balance between accuracy and precision must in practice be found, depending on the application. In accelerometry, the emphasis is entirely on precision. In our case, where an ultimate goal is to derive intrinsic stellar parameters through comparison with astrometric radial velocities, the balance is instead shifted towards accuracy. A practical consequence is that our method would be suboptimal for accelerometry. 


\section{Conclusions}

At the accuracy level $\left(\$ 100 \mathrm{~m} \mathrm{~s}^{-1}\right)$ permitted by modern spectrometers such as ELODIE it is necessary to review standard procedures for radial-velocity determination in order to produce results that have a clear physical meaning and are reproducible in an absolute sense. For instance, ambiguities of the classical radial-velocity concept have led to the IAU recommendation that accurate spectroscopic measurements should be given as "barycentric radial-velocity measures". Moreover, these measures refer to spectral features that also need to be clearly specified.

Based on observations obtained with the ELODIE spectrometer, we describe and apply a digital cross-correlation method specifically designed to meet the requirements for accurate radial-velocity measures. In particular:

1. The absolute wavelength scale of the observations is defined by the Solar Flux Atlas (Kurucz et al. 1984) and transferred to the stellar spectra via observations of the Moon (for long-term stability) and Th-Ar calibration spectra (for short-term stability).

2. A synthetic template is used for cross-correlation with the stellar spectra. For the present results the template consists of $1340 \mathrm{Fe}$ I lines placed at their laboratory wavelengths and having a Gaussian shape with $F W H M=5 \mathrm{~km} \mathrm{~s}^{-1}$. Together with the instrumental profile this defines the spectral features from which the line shifts are determined.

3. Normalisation of the extracted one-dimensional spectrum reduces the arbitrary weighting of different spectral regions (depending on stellar effective temperature and instrument characteristics) normally affecting cross-correlation techniques.

4. Observed line shifts are transformed to barycentric radialvelocity measures in accordance with a recent IAU resolution, using JPL ephemerides and formulae from Lindegren \& Dravins (2002).

Results for the Sun and 42 stars are given in Tables 1 and 2. The median random (internal) standard error for the stellar radialvelocity measures is $27 \mathrm{~m} \mathrm{~s}^{-1}$, while the external error is estimated at $\pm 120 \mathrm{~m} \mathrm{~s}^{-1}$. The main contributor to the external error is the uncertainty in the wavelength scale of the Solar Flux Atlas.

Acknowledgements. We thank the staff at Observatoire de Haute Provence and the ELODIE team for their enthusiastic help before, during, and after the observing runs, especially Alain Vin (HauteProvence) and Didier Queloz (Geneva). Principal Investigator for the ELODIE observing programme Astrometric versus spectroscopic radial velocities is Prof. D. Dravins at Lund Observatory, who kindly made the data available for this analysis and provided numerous advice and practical help. We gratefully acknowledge financial support from the Royal Physiographic Society in Lund, the Swedish Natural Science Research Council and the Swedish National Space Board.

\section{Appendix A: Precision of the spectral shift obtained by cross-correlation}

Expressions for the precision of the correlation peak location have been derived by several authors (Connes 1985;
Murdoch \& Hearnshaw 1991; Butler et al. 1996 and others). The standard error of $\hat{u}$ from Eq. (5) is here derived taking into account the synthetic template and the resampling and conditioning of the observed spectrum.

Let $I_{x, y}$ be the number of charges in pixel $(x, y)$, where $x$ is the pixel coordinate approximately aligned with the wavelength. We assume that the noise is uncorrelated between pixels and that the total variance per pixel is $I_{x, y}+r^{2}$. The first term represents the Poisson noise and the second the readout noise with rms value $r$ (for ELODIE, $r=8.5 \mathrm{e}^{-}$). One-dimensional spectral orders are extracted by means of a numerical slit of length $m \simeq 6$ pixels (Baranne et al. 1996). Resulting charge values $E_{x}$ are effectively the sums of $I_{x, y}$ across the spectral order, thus having uncorrelated noise with variance $\operatorname{Var}\left(E_{x}\right) \simeq E_{x}+m r^{2}$. The conditioning of the spectrum scales the noise by the known factor $h(\Lambda)=\mathrm{d} s_{i} / \mathrm{d} E_{x}$, where $\Lambda, i$ and $x$ are corresponding coordinates.

All error computations are in practice made on the resampled data, i.e. by summing over index $i$. However, in the resampled data the errors are no longer uncorrelated between adjacent points. We can take this into account by applying the appropriate factor. Thus, whenever the error propagation requires a sum of the quantity $q_{x}$ over the uncorrelated data points, it can be replaced by a sum over the resampled data $\left(q_{i}\right)$ according to the approximation:

$p \sum_{x} q_{x} \simeq \Delta \Lambda \sum_{i} q_{i}$.

Here $p=\langle\mathrm{d} \Lambda / \mathrm{d} x\rangle$ is the average pixel size expressed on the $\Lambda$ scale. For ELODIE, $p \simeq 1.0 \times 10^{-5}$.

In Eq. (5) the main error comes from the noise in the numerator (the denominator is the CCF curvature which has a relatively high signal-to-noise ratio). With $\delta$ denoting the error in a quantity caused by the total (Poisson and readout) noise we have

$\delta \hat{u} \simeq \frac{\frac{1}{2}\left(\delta g_{j+1}-\delta g_{j-1}\right)}{2 g_{j}-g_{j-1}-g_{j+1}} \Delta \Lambda$.

We can write the numerator $Q \equiv\left(\delta g_{j+1}-\delta g_{j-1}\right) / 2=$ $\sum_{i} \delta s_{i}\left(t_{i-j+1}-t_{i-j-1}\right) / 2 \simeq \Delta \Lambda \sum_{i} \delta s_{i}(\mathrm{~d} t / \mathrm{d} \Lambda)_{i-j} \simeq$ $p \sum_{x} \delta E_{x} h\left(\Lambda_{x}\right)(\mathrm{d} t / \mathrm{d} \Lambda)_{x^{\prime}}$, where $x^{\prime}=x-j \Delta \Lambda / p$. In the last approximation we have made use of Eq. (A.1). The variance of $Q$ is now found to be $\operatorname{Var}(Q) \simeq p^{2} \sum_{x}\left(E_{x}+m r^{2}\right) h^{2}\left(\Lambda_{x}\right)(\mathrm{d} t / \mathrm{d} \Lambda)_{x^{\prime}}^{2}$. Using Eq. (A.1) to re-write this as a sum over the resampled points we finally get

$\sigma_{\hat{u}} \simeq \frac{\left[p \Delta \Lambda \sum_{i}\left(E_{i}+m r^{2}\right) h_{i}^{2}\left(t_{i-j+1}-t_{i-j-1}\right)^{2}\right]^{1 / 2}}{2\left(2 g_{j}-g_{j-1}-g_{j+1}\right)}$.

Here $E_{i}$ and $h_{i}$ stand for $E_{x}$ and $h(\Lambda)$ interpolated to the appropriate coordinate. It is noted that the right-hand side of Eq. (A.3) is independent of $\Delta \Lambda$ as long as the latter is small enough that $t_{i+1}-t_{i-1} \simeq 2 \Delta \Lambda(\mathrm{d} t / \mathrm{d} \Lambda)_{i}$.

\section{References}

Allende Prieto, C., Asplund, M., García López, R. J., \& Lambert, D. L. 2002, ApJ, in press [astro-ph/0111055]

Allende Prieto, C., \& García López, R. J. 1998a, A\&AS, 131, 431 
Allende Prieto, C., \& García López, R. J. 1998b, A\&AS, 129, 41

Allende Prieto, C., García López, R. J., Lambert, D. L., \& Gustafsson, B. 1995, in Stellar Surface Structure, ed. K. G. Stassmeier, IAU Symp., 176, Poster Proceedings, 107

Asplund, M., Nordlund, Å., Trampedach, R., Allende Prieto, C., \& Stein, R. F. 2000, A\&A, 359, 729

Baranne, A. 1999, in IAU Coll., 170, Precise Stellar Radial Velocities, ed. C. D. Hearnshaw, \& J. B. Scarfe, ASP Conf. Ser., 185, 1

Baranne, A., Mayor, M., \& Poncet, J. L. 1979, Vistas in Astronomy, 23, 279

Baranne, A., Queloz, D., Mayor, M., et al. 1996, A\&AS, 119, 373

Barbier-Brossat, M., \& Figon, P. 2000, A\&AS, 142, 217

Beers, T. C., Chiba, M., Yoshii, Y., et al. 2000, AJ, 119, 2866

Brandt, P. N., \& Solanki, S. K. 1990, A\&A, 231, 221

Bundy, K. A., \& Marcy, G. W. 2000, PASP, 112, 1421

Butler, R. P., Marcy, G. W., Williams, E., et al. 1996, PASP, 108, 500

Chamberlin, A. B., Yeomans, D. K., Chodas, P. W., et al. 1997, BAAS, 29,1014

Connes, P. 1985, Ap\&SS, 110, 211

Deming, D., \& Plymate, C. 1994, ApJ, 426, 382

Dravins, D. 1987, A\&A, 172, 211

Dravins, D. 1999, in IAU Coll., 170, ed. J. B. Hearnshaw, \& C. D. Scarfe, Precise Stellar Radial Velocities, ASP Conf. Ser., 185, 268

Dravins, D., Gullberg, D., Lindegren, L., \& Madsen, S. 1999a, in IAU Coll., 170, ed. J. B. Hearnshaw, \& C. D. Scarfe, Precise Stellar Radial Velocities, ASP Conf. Ser., 185, 41

Dravins, D., Lindegren, L., \& Madsen, S. 1999b, A\&A, 348, 1040

Dravins, D., Lindegren, L., Madsen, S., \& Holmberg, J. 1997, ESA SP-402: Hipparcos - Venice '97, 402, 733

Dravins, D., Lindegren, L., \& Nordlund, A. 1981, A\&A, 96, 345

Dravins, D., \& Nordlund, A. 1990, A\&A, 228, 203

Evans, D. S. 1967, in Determination of radial velocities and their applications, ed. A. H. Batten, \& J. F. Heard, IAU Symp., 30, 57

Giorgini, J. D., Yeomans, D. K., Chamberlin, A. B., et al. 1996, BAAS, 28,1158

Gray, D. F. 1982, ApJ, 255, 200

Gray, D. F., \& Nagel, T. 1989, ApJ, 341, 421

Griffin, R. E. M. 1999, in IAU Coll., 170, Precise Stellar Radial Velocities, ed. J. B. Hearnshaw, \& C. D. Scarfe, ASP Conf. Ser., 185,52

Griffin, R. E. M., David, M., \& Verschueren, W. 2000, A\&AS, 147, 299

Griffin, R. F. 1967, ApJ, 148, 465

Griffin, R. F., Gunn, J. E., Zimmerman, B. A., \& Griffin, R. E. M. 1988, AJ, 96, 172

Grimmett, G. R., \& Stirzaker, D. R. 1982, Probability and random processes (Oxford: Clarendon Press)

Gullberg, D. 1999, in IAU Coll., 170, Precise Stellar Radial Velocities, ed. J. B. Hearnshaw, \& C. D. Scarfe, ASP Conf. Ser., 185, 286

Gunn, A. G., Hall, J. C., Lockwood, G. W., \& Doyle, J. G. 1996, A\&A, 305,146

Kurucz, R. L., Furenlid, I., Brault, J., \& Testerman, L. 1984, Solar flux atlas from 296 to $1300 \mathrm{~nm}$ (National Solar Observatory, Sunspot)
Latham, D. W., \& Stefanik, R. P. 1992, Trans. IAU, XXI B, 269

Lindegren, L. 1978, in ed. F. V. Prochazka, \& R. H. Tucker, IAU Coll., 48, Modern Astrometry, University Observatory Vienna, 197

Lindegren, L., \& Dravins, D. 2002, A\&A, in preparation

Lindegren, L., Dravins, D., \& Madsen, S. 1999, in IAU Coll., 170, Precise Stellar Radial Velocities, ed. J. B. Hearnshaw, \& C. D. Scarfe, ASP Conf. Ser., 185, 73

Lindegren, L., Madsen, S., \& Dravins, D. 2000, A\&A, 356, 1119

Livingston, W., Wallace, L., Huang, Y., \& Moise, E. 1999, in High Resolution Solar Physics: Theory, Observations, and Techniques, ed. T. R. Rimmele, K. S. Balasubramaniam, \& R. R. Radick, ASP Conf. Ser., 183, 494

Madsen, S., Dravins, D., \& Lindegren 2002, A\&A, 381, 446

Marcy, G. W., Butler, R. P., Williams, E., et al. 1997, ApJ, 481, 926

Mayor, M., \& Queloz, D. 1995, Nature, 378, 355

McMillan, R. S., Moore, T. L., Perry, M. L., \& Smith, P. H. 1993, ApJ, 403, 801

Morse, J. A., Mathieu, R. D., \& Levine, S. E. 1991, AJ, 101, 1495

Murdoch, K., \& Hearnshaw, J. B. 1991, Ap\&SS, 186, 137

Nadeau, D., \& Maillard, J. 1988, ApJ, 327, 321

Nave, G., Johansson, S., Learner, R. C. M., Thorne, A. P., \& Brault, J. W. 1994, ApJS, 94, 221

Nidever, D. L., Marcy, G. W., Butler, R. P., Fischer, D. A., \& Vogt, S. S. 2002, ApJ, submitted [astro-ph/0112477]

Nordström, B., Latham, D. W., Morse, J. A., et al. 1994, A\&A, 287, 338

Pallé, P. L., Jiménez, A., Pérez Hernández, F., et al. 1995, ApJ, 441, 952

Perryman, M. A. C., Brown, A. G. A., Lebreton, Y., et al. 1998, A\&A, 331,81

Queloz, D. 1996, ELODIE Guide de l'utilisateur Programme de Réduction (Observatoire de Haute-Provence, manual available on WWW)

Rickman, H. 2002, IAU Inf. Bull., 91

Skuljan, J., Hearnshaw, J. B., \& Cottrell, P. L. 2000, PASP, 112, 966

Smith, M. A., Livingston, W., \& Huang, Y. 1987, PASP, 99, 297

Soderblom, D. R., \& Mayor, M. 1993, AJ, 105, 226

Spruit, H. C., Nordlund, A., \& Title, A. M. 1990, ARA\&A, 28, 263

Stefanik, R. P., Latham, D. W., \& Torres, G. 1999, in IAU Coll., 170, Precise Stellar Radial Velocities, ed. J. B. Hearnshaw, \& C. D. Scarfe, ASP Conf. Ser., 185, 354

Tang, F., Howard, R., \& Adkins, J. M. 1984, Sol. Phys., 91, 75

Turon, C., Perryman, M. A. C., \& Priou, D. 1998, Celestia 2000 (ESA SP-1220)

Udry, S., Mayor, M., \& Queloz, D. 1999, in IAU Coll., 170, Precise Stellar Radial Velocities, ed. J. B. Hearnshaw, \& C. D. Scarfe, ASP Conf. Ser., 185, 367

Verschueren, W., David, M., \& Griffin, R. E. M. 1999, A\&AS, 140, 107

Wilson, R. E. 1953, General Catalogue Of Stellar Radial Velocities (Papers of the Mount Wilson Observatory, vol. 8) 